\title{
The Feminine Style and the Reinvention of Idols: Identifying and Guiding the Masculinity of "Generation Z" Fans
}

\author{
Yu Chen ${ }^{1, a}$, Liu Yang ${ }^{2, b^{*}}$ \\ ${ }^{1}$ Associate Professor, Department of Chinese, Xihua University, Chengdu, Sichuan, China \\ ${ }^{2}$ Graduate Student, Department of Chinese, Xihua University, Chengdu, Sichuan, China \\ a71223735@qq.com \\ b*yl17350759923@163.com
}

\begin{abstract}
In recent years, the feminine aesthetics of the male image has triggered heated discussions and image anxiety among the public. This article aims to explore the male image aesthetics of "Generation Z" fans, who are mainly teenagers, and to explore the roots of the feminine and refined aesthetics from traditional inheritance, social psychology and media culture, in order to analyse the rationality of the current discussion on "spiritual effeminacy" and national It also analyses the rationality of the current discussion on "spiritual femininity" and national image anxiety, and proposes strategies for guiding fans' aesthetics.
\end{abstract}

Keywords: Generation Z, masculine image, feminine aesthetic, fan-led

\section{阴柔之风与偶像重塑—— “Z世代” 粉丝的男性形象审 美辨析与引导 \\ 陈或 ${ }^{1, a}$ 杨柳 ${ }^{2, b *}$}

${ }^{1}$ 西华大学文学与新闻传播学院副教授, 成都, 四川, 中国

2 西华大学中文系研究生, 成都, 四川, 中国

a71223735@qq.com

b*yl17350759923@163.com

\section{摘要}

近年来，男性形象的阴柔审美引发了大众的激烈讨论和形象焦虑。本文旨在通过探索以青少年为主体的 “ $Z$ 世 代” 粉丝的男性形象审美, 从传统继承、社会心理、媒介文化多个维度挖掘阴柔精致审美的根源, 来分析当前 对于 “精神娘气” 的讨论和民族形象焦虑的合理性, 进而对粉丝审美的引导策略提出建议。

关键词: Z世代, 男性形象, 阴柔审美, 粉丝引导

\section{1. 前言}

“Z 世代”一词和 “X世代”、“Y世代”一样，最 早出现在亨特・汤普森的《比性更好:一个政治迷的 自白》中, 是一个群体概念, 泛指在 1995 年到二十 一世纪的最初十年内出生的世代。“Z 世代” 因其自
出生起就与网络时代无缝对接的特点, 又被称为 “网 生代”、“互联网世代”。“Z世代”习惯于在一个时刻 相连的世界中互动交流, 具有独特的多样性 ${ }^{[6]}$ 。“ $Z$ 世 代” 多为青少年, 他们品味独特, 风格多变, 在个体 的精神追求方面有了更高的期待, 对男性形象的审美 较传统审美也大有不同。 
2018 年 9 月 1 日, 中央一台播出的大型公益教 育节目《开学第一课》邀请了 “新 F4” 成员即官鸿、 吴希泽、梁靖康、王鹤棣开场表演, 由此引起了部分 家长和网友的不满。他们认为“新 $\mathrm{F} 4$ ”四人气质阴柔, 缺乏 “阳刚之气”, 会误导青少年, 影响青少年的性 别判断, 甚至对这四位年轻艺人进行 “娘炮羞辱”, 引发了 “少年娘, 则国娘” 的论断 ${ }^{[4]}$ 。舆情哗然, 其 本质上是在传统男性气质受到挑战的情境下的社会 性焦虑——即对青少年 “阴柔”、“娘气” 等审美倾向 的焦虑。但何为 “阴柔” ? 何为 “阳刚” ? 当传统男 性形象审美被挑战, 这种集体焦虑是否有存在的必要? 本文希望通过辨析以青少年为主体的 “Z世代” 粉丝 的男性形象审美，来考察这种集体焦虑的合理性。

\section{2. “Z世代” 粉丝的男性偶像审美倾向}

\section{$2.1 “ Z$ 世代” 粉丝的高热度男性偶像榜单}

“Z世代” 既是 “网生代”，也是 “追星” 的一代。 新浪微博明星超话与腾讯兴趣部落是 “ $Z$ 世代” 粉丝 追星两大聚集地, 新浪微博和腾讯两家的年度娱乐白 皮书中的明星排行榜涉及热搜、人气、商业价值等多 个维度的量化分析, 非常具有参考价值。根据 2018-2020 年新浪微博娱乐白皮书和腾讯娱乐白皮书 （新浪微博娱乐白皮书和腾讯娱乐白皮书属于中央 网信办 8 月份发布的《关于进一步加强 “饭圈” 乱象 治理的通知》中提出的需要取消的明星艺人榜单, 但 该排行榜具有数据参考价值, 可以用于分析粉丝构成 和明星热度) 对上榜男明星进行叠加篮选, 所得重合 名单中的男明星一定程度上代表了近三年的热度高、 流量大、人气高的男明星群体。

根据 2018 年度新浪微博粉丝白皮书、2019 年新 浪微博明星白皮书、2020 新浪微博娱乐白皮书明星 篇以及 2018-2020 年腾讯娱乐白皮书明星篇, 重叠篎 选后的男明星统计如下:

表格 1 重叠篮选后的男明星名单

\begin{tabular}{cc}
\hline \multicolumn{2}{c}{ 重叠笁选后的男明星 } \\
\hline 蔡徐坤 & 肖战 \\
邓伦 & 周震南 \\
鹿晗 & 陈坤 \\
王俊凯 & 范丞丞 \\
王一博 & 何炅 \\
王源 & 黄子搯 \\
吴亦凡 & 李易峰 \\
易烊千晧 & 罗云熙 \\
张艺兴 & 秦吴 \\
朱一龙 & 朱正廷 \\
陈立农 & 刘昊然 \\
华晨宇 & 冯绍峰 \\
黄明吴 & 管栎 \\
黄晓明 & 张云雷 \\
李现 & 王琳凯 \\
罗志祥 & 陈伟霆 \\
周杰伦 & \\
\hline
\end{tabular}

\section{2 上榜男性偶像的形貌类型化分析}

“形象”一词, 除了表示人的形貌, 还含有人的 性格特征的意思, 是包含二者在内的概念。而众人讨 论男性的 “阴柔”与 “阳刚” 时, 往往直接将其等同 于男性形象的讨论, 而他们攻击的艺人只是外表 “女 性化”, 并没有什么直接的证据证明他们行为“娘气”, 性格 “女性化”, 众人的 “娘炮羞辱” 其实有片面评 价的嫌疑。探究 “Z世代” 粉丝对上榜男性的形貌的 讨论热度, 可以用以分析 “ $Z$ 世代” 粉丝对男性偶像 的关注原因, 还可以对形貌关注度高的明星进行形貌 类型化分析，总结 “ $Z$ 世代” 粉丝的男性形貌审美特 点 $^{[2]}$ 。

哔哩哔哩网站（简称B站）是国内领先的年轻人 文化娱乐社区，根据艾瑞数据 2020 年发布的B站分析 报告中的用户性别分析可知, B站的男性用户占比 $52.92 \%$ ，女性用户占比 $47.08 \%$ ，同时满足男性和女 性的需求。根据其用户人群分析可知, 2019 年B站 30 岁以下的人群占比 $73.68 \%$, 多为 “ $\mathrm{Z}$ 世代”。B站有大 量明星粉丝对明星作品的再生产投稿, 将 “明星名字 +颜值” 作为关键词进行搜索, 搜索所得数据能反映 明星颜值的讨论热度的高低。

在B站搜索 “明星名字+颜值” 词条, 对每个明星 的相关投稿数据按大小进行排序，得到排名如下： 


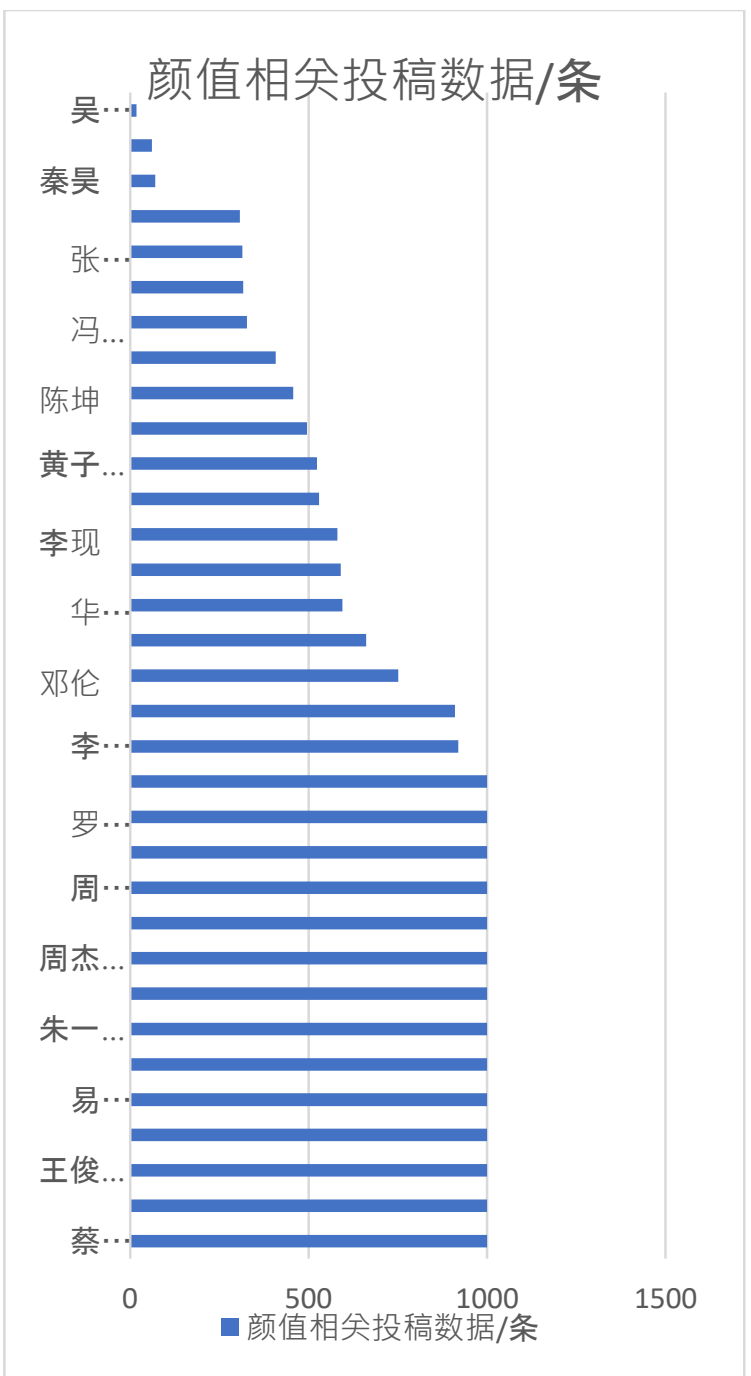

图表 1 明星的颜值相关投稿数据排序

由此去除数据低于 500 的明星, 得到颜值热度高 的明星有: 陈坤、黄晓明、黄子蹈、陈立农、李现、 陈伟霆、华晨宇、王源、邓伦、刘昊然、李易峰、王 琳凯、罗云熙、范丞丞、周震南、肖战、周杰伦、黄 明昊、朱一龙、张艺兴、易烊千秃、王一博、王俊凯、 鹿晗、蔡徐坤。（注：B站搜索结果数量最高显示为 $1000+$ )

根据这一名单, 结合粉丝评论对他们做形貌类型 化分析, 结果如下:

表格 2 上榜男性偶像的形貌类型化分析

\begin{tabular}{|c|c|c|}
\hline 形貌类型 & 特征描述 & 偶像名单 \\
\hline \multirow{4}{*}{ A. 俊美精致的贵公 } & 小头宽肩 & 蔡徐坤 \\
\cline { 3 - 3 } & 五官立体精 & 致 \\
\cline { 3 - 3 } & 打扮时尚 & 鹿晗 \\
\cline { 3 - 3 } & 模特身材 \\
\cline { 3 - 3 } & 皮肤白晳 & 范丞丞 \\
\cline { 3 - 3 } & & 黄明昊 \\
\cline { 3 - 4 } & & 王琳凯 \\
\cline { 3 - 4 } & & 黄子蹈 \\
\hline
\end{tabular}

\begin{tabular}{|c|c|c|}
\hline & & 陈立农 \\
\hline \multirow{6}{*}{ B. 青春阳光的少年 } & \multirow{6}{*}{$\begin{array}{c}\text { 皮肤白晳 } \\
\text { 五官清秀 } \\
\text { 面容幼态 } \\
\text { 青涩干净 } \\
\text { 阳光活泼 } \\
\text { 少年感 }\end{array}$} & 王俊凯 \\
\hline & & 易烊千玺 \\
\hline & & 刘昊然 \\
\hline & & 王源 \\
\hline & & 周震南 \\
\hline & & 李易峰 \\
\hline \multirow{5}{*}{$\begin{array}{c}\text { C. 温润如玉的谦谦 } \\
\text { 君子 }\end{array}$} & \multirow{5}{*}{$\begin{array}{l}\text { 高眉骨 } \\
\text { 深眼窝 } \\
\text { 浓睫毛 } \\
\text { 沉静斯文 } \\
\text { 忧郁克制 }\end{array}$} & 朱一龙 \\
\hline & & 罗云熙 \\
\hline & & 肖战 \\
\hline & & 王一博 \\
\hline & & 邓伦 \\
\hline \multirow{6}{*}{$\begin{array}{c}\text { D. 拥有 “性张力” 的 } \\
\text { 雅㾙型男 }\end{array}$} & \multirow{6}{*}{$\begin{array}{c}\text { 麦色皮肤 } \\
\text { 大叔 } \\
\text { 腹肌 } \\
\text { 年龄感 } \\
\text { 性张力 } \\
\text { 强荷尔蒙 }\end{array}$} & $\begin{array}{l}\text { 陈坤 } \\
\end{array}$ \\
\hline & & 陈伟霆 \\
\hline & & 黄晓明 \\
\hline & & 张艺兴 \\
\hline & & 周杰伦 \\
\hline & & 李现 \\
\hline
\end{tabular}

根据上榜男星的颜值讨论热度排序和形貌类型 化分析可知, “Z世代” 的男性形象审美具有多元化、 个性化的特点。“Z世代” 并非都是 “颜值至上”，在 上榜的明星中也不乏像何炅、秦吴、周杰伦这样广受 业界好评、拥有硬实力的明星。而就 “形貌审美” 来 说, “Z世代” 粉丝们并不是局限于对纯粹阴柔美的追 求, 也有部分群体喜欢有性张力的男明星。

尽管 “Z世代” 粉丝存在多元化、个性化的审美 特点, 但聚焦于他们的“形貌审美” 还是能够看到 “阴 柔精致” 的审美倾向。尤其是上榜明星中以选秀这种 更偏向于参考形貌的方式出道的男性idol，如陈立农、 蔡徐坤、黄明昊等, 具有明显的阴柔外形特征。

从颜值热度高的男性的形貌类型及其特点来看, 无论是皮肤白晳、五官立体的俊美贵公子型和温润君 子型, 还是拥有幼态的面容, 气质干净的青春阳光少 年都有着 “阴柔” 的外形特征, 而并非成熟的粗放气 息。至于最后一种类型, 即便是那些雅㾂型的偶像, 也是经过精致修饰过的性感与男性气质, 而非那种粗 犷阳刚之气。从上榜男星的年龄来看, 也是“小鲜肉” 居多，而并非 “大叔”占榜。

\section{3. 阴柔与精致审美倾向的成因溯源}

\section{1 文化审美基因: 阴阳流变、农耕经济与士 人传统}

中国传统文化中, 就存在着对男性阴柔之美的形 貌审美传统。从先秦至汉 “大而健壮” 的 “阳刚之美” 到魏晋南北朝的“肤脆骨柔”, 再到隋唐时形貌阳刚、 
体隗强健的审美风尚。从宋代细淢温和，再到元明之 际的雄壮刚健，又到清朝的白净阴柔，中国传统男性 形貌审美虽然有阳刚与阴柔的交替变迁，但总体受到 了农耕文化和士人传统的影响。农耕经济聚焦于土地 耕种，孕育出温顺柔情、对大地之母有着深厚感情的 农耕民族 ${ }^{[1]}$ 。而儒道互补、“天人合一” 的士人传统 也一直占据上风，形成了对温润如玉的男性气质的推 崇 ${ }^{[8]}$ 。

\section{2 社会心理根源: 后工业应然、自我画像与 心理代偿}

阴柔与精致的审美倾向有其社会心理根源。首先, 后工业社会的和平发展与经济腾飞使得男性的力量 特质不再像在工业社会那样作为 “刚需” 而受到欢迎 [5]。相反，信息社会下科技的发展使得偏中性的男性 气质更适应现代社会中的职业需求, 感性、细心等女 性气质更受推崇。此外, 现代社会中女性思潮的兴起, 女性的独立意识觉醒, 也使得温和体贴的男性更加受 到女性的欢迎。

再者, 对阴柔气质的推崇其实是现代人追求美的 过程中对自我进行画像的结果。对 “统治”了多年的 中国传统男性审美的解构, 是他们现代性精神的体现。 与此同时, 人们在追求美的同时也在美中寻找自身的 特质, 以求自我认同的实现。“ $Z$ 世代” 粉丝对阴柔形 貌的追求, 其实是传统男性气质与传统女性气质的协 调融合的趋势, 既体现了女性意识的觉醒, 又体现了 男性在审美中寻找对阴柔特性的自我认同心理。

现代社会的高度利益化与竞争激烈化也催生了 对温柔气质的找寻。高压文化下, 安全感的缺失致使 人们精神紧张, 疲于奔命, 而温柔中和的气质蒀含了 极大的情绪价值, 这也促进了人们对阴性之美的推崇 ${ }^{[7]}$ 。

\section{3 媒介文化影响: 形象的可消费}

这种审美倾向还受到了媒介文化的影响。消费文 化把人的身体变成消费品, 媒介文化与视觉文化又把 偶像形象变成最完美的身体形象，身体文化与身体美 学也强调身体的塑造与修饰。而偶像的外表形象就是 这样一种经过最精心的修饰和包装的消费品, 自然会 形成一种商品的精致之美。同时，当前的娱乐工业也 受到了日、韩造星模式和审美风格的影响, 使得国内 的养成系偶像也具有日韩系的柔美特征。

\section{4. 偶像重塑: 一个粉丝审美的引导策略}

男性形貌阴柔与精致的审美趋势使得当前社会 持有传统男性形貌阳刚审美的主流产生了极大的焦 虑，甚至上升到了对民族衰弱、精神危机的讨论，而 这其实是对阴柔形貌的过分解读。可以看到, “Z世代” 粉丝对男性 “阴柔” 的推崇仅仅是粉丝对男性偶像的 一种形貌审美, 这背后是一种合乎文化传统、社会潮
流的审美心理。

此外, “Z世代” 粉丝并不都是 “颜值至上”。在 2018 微博粉丝白皮书中可以看到, 专一理智型粉丝 占据 $57.7 \%$, 单一属性的粉丝不复存在。明星的公益 正能量也和粉丝热度正相关, 正能量助力明星提升了 公众好感度。粉丝关注明星的原因并不是只有其外表, 更有努力、认真、情商高、性格好等实力因素和性格 特质。即使是形貌风格本身, 也不仅仅只有 “阴柔精 致”这一种。

所以, 对于粉丝的审美应持有 “重引导而非批判” 的立场, 而不是一味地对 $Z$ 世代的审美倾向进行文化 批判和趣味否定。应当耐心发现其中的合理的成分、 具有文化价值的因子，同时警惕其中可能会存在的危 害倾向, 并加以精神气与价值观的引导, 从而形成健 康的审美文化 ${ }^{[3]}$ 。

同时，这种引导应该以偶像形象打造为中心。因 为偶像是娱乐工业、传媒文化、粉丝之间的接榫点, 青少年粉丝的情感寄托、价值观形成、审美形构等, 在很大程度上会受到偶像文化的影响。

综上，本文就如何打造优质偶像形象的问题提出 以下几点建议。

\section{1 从阴柔之美到中和之美: 打造具有中国气 质的偶像}

沿袭中国传统人物品藻的审美标准，实现对 “天 人合一”、“刚柔并济” 审美的现代化突破。对阴柔精 致的推崇应合乎 “中庸之道”, 过而太 “妖”, 抑而过 “刚”。在保留形貌阴柔特质的同时, 将偶像气质深 化成一种具有 “外柔内刚”、“刚柔并济” 这样具有东 方审美精髓的形象气质风格, 真正传承文化精髓, 打 造具有东方气质的男性偶像。

\section{2 从形貌审美到人格审美: 打造代表中国精 神的偶像}

警惕 “颜值即正义” 的倾向，主张从颜值到人格 的审美风向。粉丝文化的凝聚力极强, 正确引导粉丝 的审美风向要实现从形貌到人格的转变。要引导粉丝 推崇偶像明星的精神内涵、道德品质、价值观主张等, 就要打造具有中国精神、正能量、行为端正的偶像。 针对粉丝为偶像打投倒牛奶事件，《人民日报》这样 评论: “一个富有内涵的“星”和一群正向追求的“粉”, 共同构成文明健康的精神家园，对现代社会的娱乐生 态、文化样态才是真正的丰富。”

\section{3 从艺人形象到艺术形象: 打造拥有优秀作 品的偶像}

警惕偶像形象空心化, 引导粉丝从艺人人设聚焦 转向艺人作品聚焦。在偶像形象打造时, 注重推崇真 正的敬业精神，淡化艺人人设加成，主张用作品说话 
的艺人评判标准。让文艺圈少一点流量明星, 多一些 实力演员，少一些娱乐圈的凹人设表演，多一些打动 人心、激励青少年的艺术形象。

\section{4 从阴柔之风到多元风尚: 打造多元审美的 偶像矩阵}

尊重多元审美，警惕审美单一。打造拥有多种风 格的偶像群体, 警惕造星工厂流水作业, 致使偶像形 象单一化。防止刻板单一的审美标准对青少年的误导, 提倡尊重个性与特色, 打造本色化、特色化、自然化 的偶像, 引导多元健康的审美风尚。

\section{5. 结论}

“Z世代” 粉丝审美的阴柔与精致倾向有其传统 基因、社会心理和媒体文化根源，具有合理性。对于 “Z世代” 粉丝男性审美的阴柔倾向问题, 主流不必 过分恐慌，更不能对形貌审美上的“阴柔”进行误读， 甚至污名化。应采取引导的策略, 从偶像的形象打造 入手, 培养一批多元的、有中国特色的、代表中国精 神的正能量偶像, 以此来正 “娱乐圈” 风气, 促进青 少年形成多元健康的审美风尚。

\section{项目基金}

本文系国家自然科学基金面上项目 “数字创意产 品多业态联动开发机理及模式研究” 阶段性成果之一, 编号: 71874142 。

\section{REFERENCES}

[1] Hu Shi. (2021). Aesthetic changes of male physiognomy in ancient China and its contemporary influence. Shandong Social Science (06), 102-108

[2] Zinan Ren. (2021). Study on the online expression of Generation $Z$ and its discourse system construction (Master's thesis, Shanghai Normal University).

[3] Furong Guo \& Lianghong Wei. (2021). Typical characteristics, prominent problems and value guidance of fan behavior in social media era. Journal of Chongqing University of Posts and Telecommunications (Social Science Edition) (02), 88-96.

[4] Qi Gai. (2019). Gender temperament and aesthetic generation gap: From "sissy shaming" to "male anxiety" in current media culture. Academic Research (07), 151-155.

[5] Jingbo Xu. (2018). The thin waist: mass media, political games and the changing aesthetics of the male form in modern times. Shilin (05),
$97-107+220$.

[6] Turner, Anthony (2015). Generation Z: Technology And Social Interest. Journal of Individual Psychology. 71(2): 103-113.

[7] Xiaoling Li. (2009). A modern interpretation of spiritual crisis. Journal of Xi'an College of Arts and Sciences (Social Science Edition) (03), 76-78.

[8] Yanqin Peng \& Haosheng Ye. (2006). Personality: An interpretation of traditional Chinese aesthetic psychology. Journal of Southwest University (Humanities and Social Sciences Edition) (01), 9-14. 